\section{Mehr Depressionen und Suizide}

Diabetiker haben im Vergleich zu Gesunden eine drei- bis vierfach erhöhte Prävalenz für die Manifestation einer Depression.

- 40-80\% aller Patienten mit einer schweren rezidivierenden Depression haben Suizidgedanken.

- 20-60\% unternehmen einen Suizidversuch, der bei $15 \%$ auch zur Selbsttötung führt.

- Die Jahrespunktprävalenz für Suizid betrug in einer Längsschnittuntersuchung mit 96 erkrankten Kindern und Jugendlichen 21\%.

\section{Diabetiker glauben an sich selbst}

Leitlinien empfehlen Diabetikern die Glukoseselbstmessung zum Therapiemanagement. Doch die Realität sieht anders aus. Wie eine neue Studie zeigt, ermitteln viele Patienten ihre Werte über den Daumen gepeilt. Knapp über 70\% der Diabetiker entscheiden über ihre Medikation und ihre Insulindosierung, ohne eine vorherige Glukoseselbstmessung vorgenommen $\mathrm{zu}$ haben. Dies ergab eine Befragung von 297 erwachsenen Typ-2-Diabetikern. 77\% der Patienten gaben an, dass ihnen ihr Körper ohne Test mitteilen würde, ob der Blutzuckerspiegel zu niedrig oder zu hoch ist.

Media Round Table, EASD-Kongress, Berlin, Oktober 2012

\section{Flexibel bleiben ...}

... ist bei der Behandlung von Typ-2-Diabetes besonders wichtig. Denn auch wenn eine Insulintherapie unvermeidbar wird, sollten Sie die Wünsche, Fähigkeiten und Komorbiditäten Ihrer Patienten berücksichtigen. Sogar die deutschen Fachgesellschaften unterstreichen in ihrer Nationalen Versorgungsleitlinie 2013 die Notwendigkeit von individuellen Therapiezielen und geben einen „HbAlc-Korridor" von $6,5-7,5 \%$ an. Mehr dazu und viele andere spannende Themen finden Sie in unserem Schwerpunkt.

\section{Claudia Daniels} Redakteurin

\title{
Süßes Vergessen: Diabetiker erwischt die Demenz zwei Jahre früher
}

Nach einer bevölkerungsbasierten Studie aus Australien entwickelt sich eine vaskuläre Demenz im Schnitt fast drei Jahre und eine Alzheimer-Demenz gut ein Jahr früher als bei stoffwechselgesunden Personen. Bei 17,3\% aller Patienten bestand vor der Demenzerkrankung ein Diabetes. Von den Patienten, die schon vor dem 75. Lebensjahr eine Demenz entwickelt hatten, war sogar fast jeder Vierte diabeteskrank. Insgesamt waren Diabetiker im Durchschnitt 2,2 Jahre jünger, wenn die Demenz festgestellt wurde. Die Assoziation zwischen
Diabetes und frühem kognitivem Verlust blieb auch dann erhalten, wenn andere Risikofaktoren für eine Demenz berücksichtigt wurden. Das Risiko korrelierte mit der Dauer des Diabetes und war deutlicher ausgeprägt bei der vaskulären Demenz: Sie trat bei Studienteilnehmern, die seit mindestens 15 Jahren an Diabetes litten, im Mittel 5,7 Jahre früher auf als bei Studienteilnehmern ohne Diabetes.

(bs)

Zilkens RR et al, American Journal of Epidemiology Advance Access (online first)

\section{Diabetes durch Stress im Job?}

Nimmt der berufliche Stress überhand, kann bei Frauen der Zuckerstoffwechsel entgleisen, so eine aktuelle schwedische Untersuchung. Die knapp 5500 Teilnehmer waren zu Studienbeginn zwischen 36 und 56 Jahre alt und gemäß oralem Glukosetoleranztest gesund. Nach acht bis zehn Jahren waren 60 Frauen und 111 Männer an Typ-2-Diabetes erkrankt. Ein geringer Entscheidungsspielraum etwa erhöhte bei Frauen das Diabetesrisiko um das Zweifache (OR 2,4; 95\% CI 1,1-5,2). Um das Vierfache stieg es, wenn zum geringen Entscheidungsspielraum eine hohe
Arbeitsbelastung hinzukam (OR 4,2; 95\% CI 2,0-8,7). Männer hingegen scheinen nicht Gefahr zu laufen, aufgrund ihres beruflichen Stresses später an Diabetes zu erkranken. Vielmehr sank ihr Diabetesrisiko mit hoher Arbeitsanforderung und -belastung (OR 0,5; 95\%CI 0,3-0,9). Auch Männer, die sich bei hoher Arbeitsbelastung über einen großen Gestaltungsfreiraum freuen konnten, erkrankten seltener (OR 0,4; 95\% CI 0,2-0,9).

\section{(dk)}

Eriksson AK et al, Diabetes Care 2013,

(online first)

\section{Herzklopfen kündigt Zucker an}

Männer, deren Herzen in Ruhe überdurchschnittlich schnell schlagen, haben einer australischen Studie* zufolge ein erhöhtes Risiko, Diabetes zu entwickeln. Der kausale Zusammenhang dieses Phänomens bleibt jedoch ungeklärt.

Von knapp 6000 Männern und Frauen einer australischen Studie erkrankten 221 innerhalb von fünf Jahren 221 an Diabetes mellitus. Dabei waren hohe Ruheherzfrequenzen eindeutig mit dem Erkrankungsrisiko assoziiert: Ab 80 Schlägen pro Minute war das Diabetesrisiko um den Faktor 1,89 gegenüber Personen mit langsamerem Herzschlag ( $<60$ pro Minute) erhöht.

Noch viel deutlicher wurde der Zusammenhang, wenn man nur die normal- oder leicht übergewichtigen Männer mit einem BMI von $<30 \mathrm{~kg} / \mathrm{m}^{2}$ betrachtete: Bei ihnen stieg das Risiko ab einer Herzfrequenz von 80 pro $\mathrm{Mi}$ nute um mehr als das Fünffache. Dagegen bestand weder für Frauen noch für adipöse Männer eine übermäßige Diabetesgefahr.
*The Australian Diabetes Obesity and Lifestyle (AusDiab) Study,

Grantham NM et al, Diabet Med 2013,

30: $421-427$ 